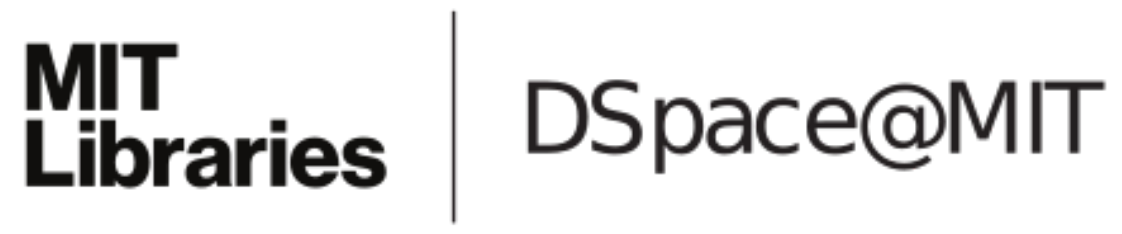

\author{
MIT Open Access Articles
}

Topological Transition in a Non-Hermitian Quantum Walk

The MIT Faculty has made this article openly available. Please share how this access benefits you. Your story matters.

Citation: Rudner, M. S., and L. S. Levitov. "Topological Transition in a Non-Hermitian Quantum Walk." Physical Review Letters 102.6 (2009): 065703. (C) 2010 The American Physical Society.

As Published: http://dx.doi.org/10.1103/PhysRevLett.102.065703

Publisher: American Physical Society

Persistent URL: http://hdl.handle.net/1721.1/51071

Version: Final published version: final published article, as it appeared in a journal, conference proceedings, or other formally published context

Terms of Use: Article is made available in accordance with the publisher's policy and may be subject to US copyright law. Please refer to the publisher's site for terms of use. 


\title{
Topological Transition in a Non-Hermitian Quantum Walk
}

\author{
M. S. Rudner ${ }^{1,2}$ and L. S. Levitov ${ }^{1,3}$ \\ ${ }^{1}$ Department of Physics, Massachusetts Institute of Technology, 77 Massachusetts Avenue, Cambridge, Massachusetts 02139, USA \\ ${ }^{2}$ Department of Physics, Harvard University, 17 Oxford Street, Cambridge, Massachusetts 02138, USA \\ ${ }^{3}$ Kavli Institute for Theoretical Physics, University of California, Santa Barbara, California 93106, USA \\ (Received 13 July 2008; revised manuscript received 26 November 2008; published 12 February 2009)
}

\begin{abstract}
We analyze a quantum walk on a bipartite one-dimensional lattice, in which the particle can decay whenever it visits one of the two sublattices. The corresponding non-Hermitian tight-binding problem with a complex potential for the decaying sites exhibits two different phases, distinguished by a winding number defined in terms of the Bloch eigenstates in the Brillouin zone. We find that the mean displacement of a particle initially localized on one of the nondecaying sites can be expressed in terms of the winding number, and is therefore quantized as an integer, changing from zero to one at the critical point. We show that the topological transition is relevant for a variety of experimental settings. The quantized behavior can be used to distinguish coherent from incoherent dynamics.
\end{abstract}

The recent achievement of quantum control in superconducting artificial atoms coupled to microwave resonators opens many new possibilities to investigate coherent dynamics in many-level quantum systems using solid-state devices [1]. These systems, as well as those realized in phase qubits [2], feature dynamics on one or several ladders of discrete quantum states [1]. Strong coherent coupling of these states to external ac fields and to each other can be used as a vehicle to generate single photons [3], to realize quantum state storage and transfer [4,5], and to demonstrate nonlinear quantum-optical phenomena $[6,7]$.

In light of these new possibilities, it is tempting to look for new phenomena that could serve as a litmus test of quantumness in ladders of quantum states. Here we discuss an example of a quantum walk that exhibits a topological transition resulting in a discontinuous behavior of observables which is absent for an incoherent classical walk.

A quantum system is said to exhibit a topological transition when it features several phases, characterized by a topological invariant that takes on different quantized values in each of these phases [8]. Topological transitions are of special interest in part because they are often robust against many types of noise [9], as is well known, for example, in the case of the quantized Hall effect.

We consider a quantum walk on a bipartite onedimensional (1D) lattice, from which the "walker" (particle) can decay whenever it resides on the sites of one of the sublattices [see Fig. 1(a)]. Because of hopping between sites, a particle initially localized on any of the nondecaying sites will eventually decay from the system. Surprisingly, we find that the average displacement of the particle before its decay, $\langle\Delta m\rangle=\sum_{m} m P_{m}$, is exactly quantized as an integer ( 0 or 1 unit cells), where $P_{m}$ is the probability distribution for decay from different sites [see Figs. 1(b) and 1(c)].

This quantization results from an underlying topological structure; in this case it is the winding number of the relative phase between two components of the Bloch wave function, shown in Fig. 1(d). Using the topological origin of this phenomenon, we are able to show that the quantization is insensitive to parameters and is robust against certain types of noise and decoherence. This quantized behavior should be contrasted with the continuous behavior of $\langle\Delta m\rangle$ in the case when hopping is completely incoherent, shown in Fig. 1(c).

In our discussion, we first focus on the general aspects of the topological transition, after which we outline its relation to a Jaynes-Cummings model with decay. Models of
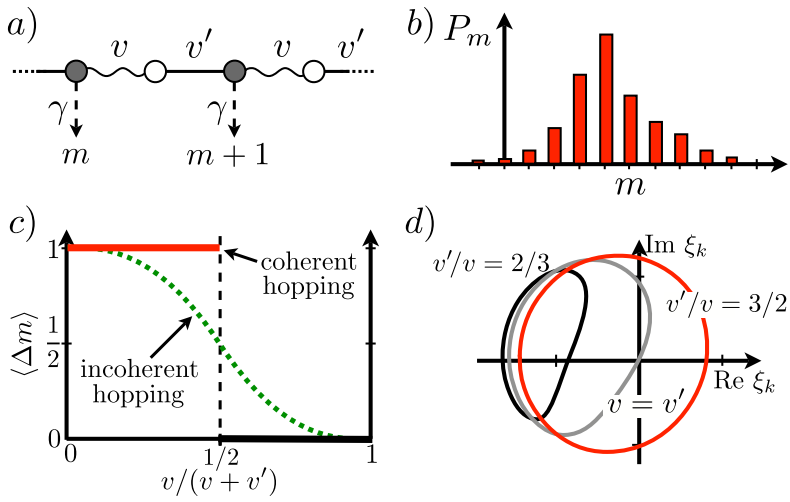

FIG. 1 (color online). (a) Quantum walk on a bipartite 1D lattice. Each unit cell $m$ contains two sites $B$ (filled circles) and $A$ (open circles). Decay with rate $\gamma$ occurs from each site of the $B$ sublattice. Intracell (wavy lines) and intercell (straight lines) tunneling occur with amplitudes $v$ and $v^{\prime}$, respectively. (b) Schematic distribution of local decay probabilities $\left\{P_{m}\right\}$ used to calculate (c) the expected displacement, Eq. (3), after full decay of the initially localized state. In the absence of quantum coherence, $\langle\Delta m\rangle$ would depend smoothly on $v$ and $v^{\prime}$ (dashed green curve). The quantization of $\langle\Delta m\rangle$ is topological in nature, and is linked to (d) the winding of the Bloch eigenstates of the Hamiltonian (4). Here we plot the component ratio $\xi_{k}=\psi_{k}^{B} / \psi_{k}^{A}$ vs. momentum $k$ for $-\pi<k<\pi$. 
this type often arise in a variety of experimental systems such as trapped ions, cavity QED, and, in particular, solidstate artificial atoms coupled to resonators [1,3-5,7]. In addition, a real-space realization of our quantum walk may help settle a long standing question about the quantum coherence of vortex transport in Josephson arrays [10].

The configuration space of the problem consists of states labeled by the lattice index $m$, taking integer values $-\infty<$ $m<\infty$, and the sublattice indices $A$ and $B$. In this basis, the state of the system $|\psi\rangle$ is described by the amplitudes $\psi_{m}^{A}=\langle m A \mid \psi\rangle$ and $\psi_{m}^{B}=\langle m B \mid \psi\rangle$, and evolves according to the equations of motion

$$
\begin{aligned}
i \hbar \dot{\psi}_{m}^{A} & =\varepsilon_{A} \psi_{m}^{A}+v \psi_{m}^{B}+v^{\prime} \psi_{m+1}^{B} \\
i \hbar \dot{\psi}_{m}^{B} & =\tilde{\varepsilon}_{B} \psi_{m}^{B}+v \psi_{m}^{A}+v^{\prime} \psi_{m-1}^{A} .
\end{aligned}
$$

Without loss of generality, we choose $v>0$ and $v^{\prime}>0$. The on site energy $\tilde{\varepsilon}_{B}=\varepsilon_{B}-i \hbar \gamma / 2$ for the $B$ states has an imaginary part that accounts for the decay of these states with rate $\gamma$, while the on site energy $\varepsilon_{A}$ is real.

The problem (1) is an example of non-Hermitian quantum mechanics in one dimension, which in recent years has found applications to a variety of different problems (see Ref. [11] and references therein). Much of the interest in these 1D problems was triggered by the idea that an Anderson localization transition can occur in disordered transport with an imaginary vector potential [12]. In contrast, our problem is translationally invariant; the transition results from competition between two processes, intracell and intercell hopping, which occur with amplitudes $v$ and $v^{\prime}$ [see Fig. 1(a)].

Now, suppose the system is initialized to the $A$ state

$$
\psi_{m}^{A}=\delta_{m, 0}, \quad \psi_{m}^{B}=0
$$

at time $t=0$, and allowed to evolve freely under the equations of motion (1). Because of translational invariance, we can equivalently start anywhere on the $A$ sublattice. Under the dynamics (1), the wave packet describing the quantum walker spreads throughout the lattice and leaks out through its components on the $B$ sites, decaying completely as $t \rightarrow \infty$. Given the ability to detect the site $m$ from which the decay occurs, and thereby measure the decay probability distribution $P_{m}$ [see Fig. 1(b)], we would like to find the expected displacement

$$
\langle\Delta m\rangle \equiv \sum_{m} m P_{m}, \quad P_{m}=\int_{0}^{\infty} \gamma\left|\psi_{m}^{B}(t)\right|^{2} d t .
$$

Although $\langle\Delta m\rangle$ can be obtained from an explicit calculation of the system's time evolution operator, here we pursue a less direct approach that helps uncover the topological structure behind the solution. The result is supported by numerical simulations, which also allow us to test the model's robustness against decoherence.

As a first step in the calculation of $\langle\Delta m\rangle$, we note that the norm of a quantum state $|\psi\rangle$ evolves according to $\frac{d}{d t}\langle\psi \mid \psi\rangle=i\left\langle\psi\left|\left(\hat{H}^{\dagger}-\hat{H}\right)\right| \psi\right\rangle$. For Hermitian systems, $\hat{H}^{\dagger}=\hat{H}$ and $\frac{d}{d t}\langle\psi \mid \psi\rangle=0$. However, our system is non-
Hermitian due to the complex energy $\tilde{\varepsilon}_{B}$, and, as seen from the equations of motion (1), decays according to $\frac{d}{d t}\langle\psi \mid \psi\rangle=-\sum_{m} \gamma\left|\psi_{m}^{B}\right|^{2}$. The decay is thus described as a sum over local terms accounting for the decay from each site of the lattice, Eq. (3), with $\sum_{m} P_{m}=1$.

It is beneficial to pass to the momentum representation, $\psi_{m}^{B}=\frac{1}{2 \pi} \oint d k e^{i k m} \psi_{k}^{B}$, where the integral is taken over the Brillouin zone $-\pi \leq k<\pi$. Because of the translational invariance of the system (1), the equations of motion in the Fourier representation break up into $2 \times 2$ blocks, one for each value of momentum $k$ :

$$
i \hbar \frac{d}{d t}\left(\begin{array}{c}
\psi_{k}^{A} \\
\psi_{k}^{B}
\end{array}\right)=\left(\begin{array}{cc}
\varepsilon_{A} & v_{k} \\
v_{k}^{*} & \tilde{\varepsilon}_{B}
\end{array}\right)\left(\begin{array}{l}
\psi_{k}^{A} \\
\psi_{k}^{B}
\end{array}\right),
$$

with $v_{k}=v+v^{\prime} e^{i k}$. The two-component wave functions for different $k$ values are decoupled. The probability density $p_{k}(t) \equiv\left|\psi_{k}^{A}(t)\right|^{2}+\left|\psi_{k}^{B}(t)\right|^{2}$ to find the system with momentum $k$ at time $t$ decays as $\partial_{t} p_{k}=-\gamma\left|\psi_{k}^{B}(t)\right|^{2}$.

Writing $m$ as a derivative with respect to $k$ via $m \psi_{m}^{B}=$ $-\frac{i}{2 \pi} \oint d k \frac{d}{d k}\left(e^{i k m}\right) \psi_{k}^{B}$ and integrating by parts to move the derivative onto $\psi_{k}^{B}$, we bring Eq. (3) to the form

$$
\langle\Delta m\rangle=i \gamma \int_{0}^{\infty} d t \oint \frac{d k}{2 \pi} \psi_{k}^{B *} \frac{\partial \psi_{k}^{B}}{\partial k} .
$$

Next, we use the polar decomposition $\psi_{k}^{B}(t)=u_{k}(t) e^{i \theta_{k}(t)}$, where $u_{k}=\left|\psi_{k}^{B}(t)\right|$ and $\theta_{k}=\arg \left\{\psi_{k}^{B}(t)\right\}$. We assume that $u_{k}(t)>0$ for all $t>0$, which follows from Eq. (4) after some algebra [13]. Using the fact that $\oint d k u_{k} \partial_{k} u_{k}=0$ is an integral of a total derivative over a closed contour, we rewrite Eq. (5) as

$$
\langle\Delta m\rangle=\oint \frac{d k}{2 \pi} \int_{0}^{\infty} d t \frac{\partial p_{k}}{\partial t} \frac{\partial \theta_{k}}{\partial k},
$$

where we replaced $-\gamma\left|u_{k}(t)\right|^{2}$ by $\partial_{t} p_{k}$ in Eq. (6). With the help of integration by parts in the integral over $t$, the time derivative can be moved from $p_{k}$ onto $\partial_{k} \theta_{k}$, giving $\langle\Delta m\rangle=I_{0}-\int_{0}^{\infty} d t \oint \frac{d k}{2 \pi} p_{k} \partial_{t}\left(\partial_{k} \theta_{k}\right)$, with

$$
I_{0}=\oint \frac{d k}{2 \pi}\left[\left.p_{k} \frac{\partial \theta_{k}}{\partial k}\right|_{t=0} ^{\infty}\right] \text {. }
$$

We will now show that the boundary term $I_{0}$ provides the only nonzero contribution to the integral (6). First, we use integration by parts on the integral over $k$ to obtain

$$
-\int_{0}^{\infty} d t \oint d k p_{k} \frac{\partial^{2} \theta_{k}}{\partial t \partial k}=\int_{0}^{\infty} d t \oint d k \frac{\partial p_{k}}{\partial k} \frac{\partial \theta_{k}}{\partial t} .
$$

As demonstrated below, this integral vanishes because $p_{k}$ and $\partial_{t} \theta_{k}$ are both even functions of $k$.

In order to see that $p_{k}$ and $\partial_{t} \theta_{k}$ are even functions, it is helpful to view the evolution (4) within each $2 \times 2 k$ subspace as the precession of a decaying pseudospin in a (complex) magnetic field with $z$ component $\varepsilon_{A}-\tilde{\varepsilon}_{B}$ and transverse component of magnitude $2\left|v_{k}\right|=2\left|v+v^{\prime} e^{i k}\right|$. Because $\left|v_{k}\right|=\left|v_{-k}\right|$, a static rotation about the $z$ axis maps $\hat{H}_{-k}$ into $\hat{H}_{k}$, with $\hat{H}_{k}$ the $2 \times 2$ matrix in Eq. (4):

$$
e^{-i \varphi_{k} \hat{\sigma}^{z}} \hat{H}_{-k} e^{i \varphi_{k} \hat{\sigma}^{z}}=\hat{H}_{k}, \quad \varphi_{k}=\arg \left\{v_{k}\right\} .
$$


Given that the initial state (2) is oriented along the $z$ axis in pseudospin space for all $k$, in the rotated frame (9) the pseudospins associated with momenta $k$ and $-k$ evolve identically. Because state (2) has equal magnitude in all momentum sectors, the moduli of the $k$ and $-k$ pseudospins are equal for all times, $p_{k}(t)=p_{-k}(t)$. Furthermore, from (9) their phase difference is time independent, $\theta_{-k}=$ $\theta_{k}-2 \varphi_{k}$, which proves the claim.

To evaluate $I_{0}$, we use the facts that all $k$ states are initially occupied with equal probability $p_{k}(t=0)=1$, and that the state decays completely, $p_{k}(t \rightarrow \infty)=0$. Substituting these values in Eq. (7), we find

$$
\langle\Delta m\rangle=-\oint \frac{d k}{2 \pi} \frac{\partial \theta_{k}^{0}}{\partial k}, \quad \theta_{k}^{0} \equiv \lim _{t \rightarrow 0^{+}} \theta_{k}(t) .
$$

Although $u_{k}(0)=0$, the limit $t \rightarrow 0^{+}$is well defined.

Expression (10) is a surprising result: the expected displacement of the particle as it spreads out and decays is equal to the winding number of the relative phase between components of the Bloch wave function. In particular, this means that $\langle\Delta m\rangle$ can only take on integer values. Using $\psi_{k}^{B}(d t)=-i v_{k}^{*} d t / \hbar$, we have $\theta_{k}^{0}=\arg \left\{-i v_{k}^{*}\right\}$. It is thus immediately clear that there are two possible situations depending on whether or not $v_{k}=v+v^{\prime} e^{i k}$ wraps the origin as $k$ is taken around the Brillouin zone: $\langle\Delta m\rangle=$ 1(0) when $v^{\prime}>v\left(v>v^{\prime}\right)$.

It is perhaps not entirely obvious from this discussion that the transition at $v=v^{\prime}$ is a characteristic of the Hamiltonian rather than of the initial state. To clarify this point, we examine the eigenstates of $\hat{H}_{k}$ and plot the ratio of their components $\xi_{k}=\psi_{k}^{B} / \psi_{k}^{A}$ in the complex plane. As shown in Fig. 1(d), the winding number about the origin changes from 1 at $v^{\prime}>v$ to 0 at $v^{\prime}<v$.

Furthermore, one of the eigenvalues of $\hat{H}_{k}$ becomes real at the transition $v=v^{\prime}$, where $v_{k=\pi}=0$. This indicates the formation of a nondecaying dark state with $\psi_{k=\pi}^{B}=0$; under these conditions, the $k=\pi$ component of the initial state remains stuck on the $A$ sublattice for all $t$.

Because of dark state formation, the average decay time

$$
\bar{\tau}=-\int_{0}^{\infty} t \frac{d}{d t}\langle\psi \mid \psi\rangle d t=\oint \frac{d k}{2 \pi} \int_{0}^{\infty} p_{k}(t) d t
$$

may become very long near the transition (here we used $\frac{d}{d t}\langle\psi \mid \psi\rangle=\frac{1}{2 \pi} \oint d k \partial_{t} p_{k}$ and integrated by parts). Close to the transition $v=v^{\prime}$, when $\left|v_{k \approx \pi}\right| \ll \hbar \gamma$, the dynamics (4) yields $p_{k}(t) \approx \exp \left(-\Gamma_{k} t\right)$, where $\Gamma_{k}$ is given by Fermi's golden rule: $\Gamma_{k}=\left|v_{k}\right|^{2} \gamma /\left[\left(\varepsilon_{A}-\varepsilon_{B}\right)^{2}+(\hbar \gamma / 2)^{2}\right]$. Substituting these expressions into Eq. (11), and using the change of variables $z=e^{i k}$, we get

$$
\bar{\tau}=\frac{\left(\varepsilon_{A}-\varepsilon_{B}\right)^{2}+(\hbar \gamma / 2)^{2}}{2 \pi i \gamma} \oint \frac{d z}{\left(v z+v^{\prime}\right)\left(v+v^{\prime} z\right)},
$$

where the integral is taken over the unit circle $|z|=1$.
Using the residue theorem, we see that the decay time indeed diverges at $v^{\prime}=v$ as $\bar{\tau} \propto 1 /\left|v-v^{\prime}\right|$.

Conspicuously, neither the quantization of $\langle\Delta m\rangle$ nor the discontinuity at $v=v^{\prime}$ depend on the values of the decay rate $\gamma$ or the energies $\varepsilon_{A / B}$. Furthermore, the analysis leading up to Eq. (10) goes through even for timedependent $\gamma$ and $\varepsilon_{A / B}$. In particular, the integral (8) still vanishes because the states $k$ and $-k$ see identical timedependent effective fields, up to a rotation (9). This suggests that the sharp transition [Fig. 1(c)] survives dephasing due to classical noise on the energy levels $\varepsilon_{A / B}$.

To investigate this remarkable indifference to dephasing, we have performed direct numerical simulations of the equations of motion (1) up to a fixed time $T$ and restricted to a finite chain of 51 unit cells. During each time step $t_{n}<$ $t<t_{n}+\Delta t$, we evolve the state forward in time and bin the probability of decay from each unit cell. In Fig. 2(a) we show the results for the mean displacement (10) and the decay time $\bar{\tau}$, obtained using the distribution $P_{m}$ [see Fig. 2(b)], and the formula $\bar{\tau}=\sum_{n}\left|\psi\left(t_{n}\right)\right|^{2} \Delta t$. The simulation, showing clear quantization, was then altered to include exponential damping of the $A-B$ off diagonal elements of the system's density matrix with rate $\Gamma_{2}$.
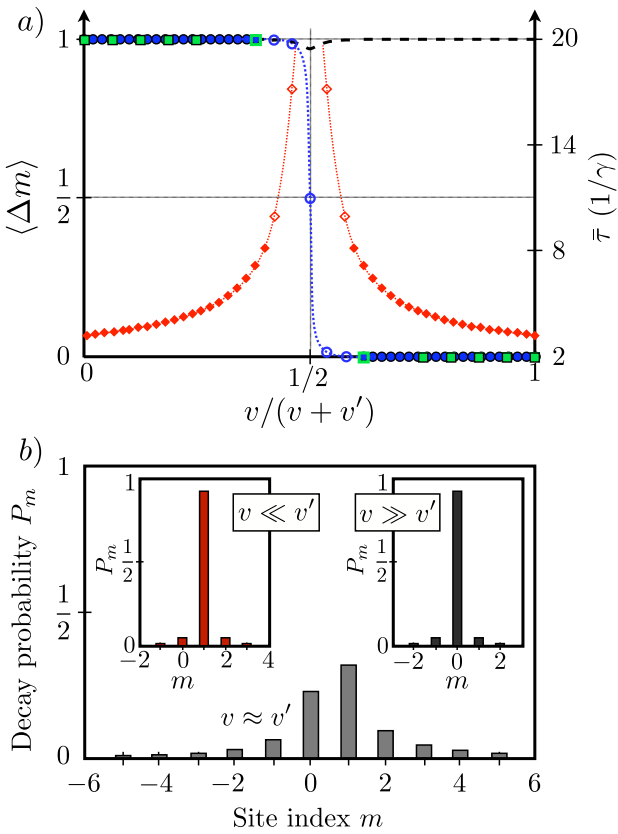

FIG. 2 (color online). Results of simulation on finite system of $N=51$ unit cells and $\gamma=\varepsilon_{A}-\varepsilon_{B}=1$. (a) Displacement $\langle\Delta m\rangle$ (blue circles) and decay time $\bar{\tau}$ (red diamonds). Filled symbols were obtained by evolving the wave function up to time $T=$ 100. Longer running time is needed near the critical point (open symbols, $T=500$ ). The black dashed line shows $1-|\psi(T)|^{2}$, used to monitor completion of the simulation. Finite length and running time effects appear as rounding of the step. Results of simulation with $\Gamma_{2}=10$ (green boxes) show that quantization survives $A-B$ decoherence. (b) Decay probabilities $\left\{P_{m}\right\}$ at $v / v^{\prime}=\frac{1}{9}, 0.85,9$. The distribution is broad near the transition $v=v^{\prime}$, but the mean remains quantized. 

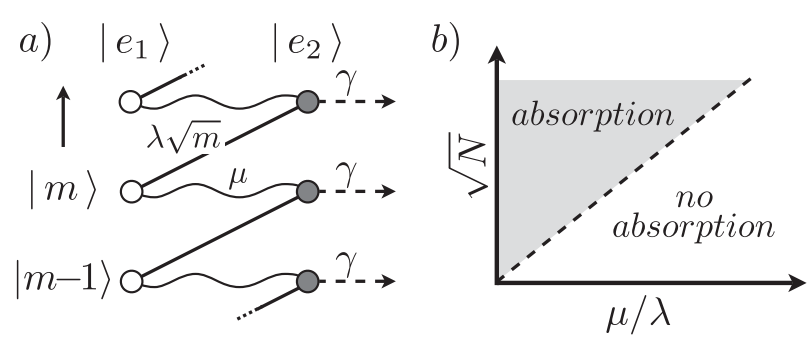

FIG. 3. Realizations of the non-Hermitian quantum walk in Jaynes-Cummings-like systems. (a) Representation of the quantum walk (1) in a form that reveals the relation to the JaynesCummings ladder of states. (b) 2D plot showing the phases of absorption and nonabsorption by the oscillator, separated by the topological transition (10). The location of the transition depends on the excitation state of the oscillator due to the $\sqrt{N}$ dependence of the harmonic oscillator matrix elements. Fluorescence is suppressed at the transition (dashed line) due to dark state formation.

Consistent with expectations, the quantization is robust against such intersublattice dephasing (green boxes).

In order to identify physical systems that realize the quantum walk studied above, it is helpful to redraw the quantum walk schematic [Fig. 1(a)] as shown in Fig. 3(a). Here the tensor product structure of the Hilbert space $\operatorname{span}\left\{|m\rangle \otimes\left|e_{1} / e_{2}\right\rangle\right\}$ is clearly displayed. This bipartite structure commonly arises in the description of a two-level system coupled to a harmonic oscillator, as in the JaynesCummings (JC) model. A similar structure arises in the problem of coupled electron and nuclear spins in quantum dots in the presence of competing spin-orbit and hyperfine interactions [14].

The JC model we consider is governed by a Hamiltonian with the general form: $\hat{H}_{\mathrm{JC}}=\hbar \Omega \hat{a}^{\dagger} \hat{a}+\frac{1}{2} \Delta\left(\hat{\sigma}^{z}-1\right)+$ $\left(\lambda e^{i \omega t} \hat{\sigma}^{+} \hat{a}+\mu e^{i \omega^{\prime} t} \hat{\sigma}^{+}+\right.$H.c. $)$, where $\hat{a}^{\dagger}$ and $\hat{a}$ are the harmonic oscillator creation and annihilation operators, $\left\{\hat{\sigma}^{\alpha}\right\}$ are Pauli matrices acting on the two-level-system subspace, and $\lambda$ and $\mu$ are parameters that control the intracell and intercell hopping $v$ and $v^{\prime}$ in the related quantum walk. The energy $\Delta$ includes the real energy difference between the states $\left|e_{1}\right\rangle$ and $\left|e_{2}\right\rangle$ and an imaginary part $-i \gamma / 2$ responsible for the decay of $\left|e_{2}\right\rangle$. Decay of this form has been studied in a variety of experimentally relevant contexts (see, e.g., Ref. [15]).

The time-dependent couplings in the general Hamiltonian allow for the possibility of external driving; depending on the specific implementation, these terms may be static or time dependent. However, in a rotating frame where $\sigma^{+}=e^{i \omega^{\prime} t} \hat{\sigma}^{+}$and $\tilde{a}=e^{i\left(\omega+\omega^{\prime}\right) t} \hat{a}$, with the two-photon resonance condition $\Omega=\omega+\omega^{\prime}$, the Hamiltonian $\hat{H}_{\mathrm{JC}}$ is time independent:

$$
H_{\mathrm{JC}}=\frac{1}{2} \Delta \varepsilon \sigma^{z}+\lambda\left(\sigma^{+} \tilde{a}+\sigma^{-} \tilde{a}^{\dagger}\right)+\mu\left(\sigma^{+}+\sigma^{-}\right),
$$

where $\Delta \varepsilon=\Delta-\omega^{\prime}$. Next, we rewrite $\tilde{a}=$ $\sum_{m>0} \sqrt{m}|m-1\rangle\langle m|$, and similarly for $\tilde{a}^{\dagger}$. In the semiclassical limit of a highly excited oscillator, $\langle m\rangle=N \gg 1$, we extend the sums to include $-\infty<m<\infty$ and replace the factors of $\sqrt{m}$ by $\sqrt{N}$, to obtain $\hat{H}_{\mathrm{JC}}=\frac{1}{2} \Delta \varepsilon \sigma^{z}+$ $\lambda \sqrt{N} \sum_{m}\left(|m-1\rangle\left\langle m\left|\sigma^{+}+\sigma^{-}\right| m\right\rangle\langle m-1|\right)+\mu\left(\sigma^{+}+\sigma^{-}\right)$. This model is equivalent to Eq. (1) with $v=\mu$ and $v^{\prime}=$ $\sqrt{N} \lambda$.

Experimentally, for the JC systems considered here, the quantity $\langle\Delta m\rangle$ is related to absorption by the oscillator. The quantization causes an abrupt change from absorption to no absorption as a function of the parameters $\lambda$ and $\mu$. Because the intercell hopping amplitude $v^{\prime}=\lambda \sqrt{N}$, the location of the transition depends on the oscillator excitation $N$ [see Fig. 3(b)]. At the transition, we predict a suppression of fluorescence by the two-level system due to dark state formation (cf. Refs. [16,17]).

In summary, the topological transition in a quantum walk problem provides a unique signature of quantum coherent dynamics. It can be used to probe quantumness in a variety of systems, including Jaynes-Cummings-type ladders of quantum states, as in qubits or two-level systems coupled to resonators [1], or grids in position space, such as Josephson arrays [10].

We thank B. I. Halperin, J. Krich, and Y. Nakamura for helpful discussions, and acknowledge support from W. M. Keck Foundation Center for Extreme Quantum Information Theory, from the National Science Foundation Graduate Research program (M.R.), and from the NSF Grant No. PHY05-51164 (L. L.).

[1] A. Wallraff et al., Nature (London) 431, 162 (2004).

[2] M. H. Devoret and J. M. Martinis, Quant. Info. Proc. 3, 163 (2004).

[3] A. A. Houck et al., Nature (London) 449, 328 (2007).

[4] M. A. Sillanpää, J. I. Park, and R. W. Simmonds, Nature (London) 449, 438 (2007).

[5] J. Majer et al., Nature (London) 449, 443 (2007).

[6] D. A. Rodrigues, J. Imbers, and A. D. Armour, Phys. Rev. Lett. 98, 067204 (2007).

[7] O. Astafiev et al., Nature (London) 449, 588 (2007).

[8] Eduardo Fradkin, Field Theories of Condensed Matter Systems (Addison-Wesley, Reading, Massachusetts, 1991).

[9] A. Kitaev, Ann. Phys. (N.Y.) 303, 2 (2003).

[10] A. van Oudenaarden, S. J. K. Várdy, and J. E. Mooij, Phys. Rev. Lett. 77, 4257 (1996).

[11] J. Feinberg and A. Zee, Nucl. Phys. B504, 579 (1997).

[12] N. Hatano and D. R. Nelson, Phys. Rev. Lett. 77, 570 (1996); Phys. Rev. B 56, 8651 (1997).

[13] An explicit form of the evolution operator, found from Eq. (4), gives $\psi_{k}^{B}(t)$ which is nonzero at all $t>0$ except when both $\varepsilon_{A}=\varepsilon_{B}$ and $\frac{1}{4} \gamma<\left|v_{k}\right|$.

[14] M. S. Rudner and L. S. Levitov, arXiv:0807.2048v1.

[15] P. M. Radmore and P. L. Knight, J. Phys. B 15, 561 (1982).

[16] P. M. Alsing, D. A. Cardimona, and H. J. Carmichael, Phys. Rev. A 45, 1793 (1992).

[17] J. I. Cirac, A. S. Parkins, R. Blatt, and P. Zoller, Phys. Rev. Lett. 70, 556 (1993). 